\title{
Optical coherence tomography to evaluate the interaction of different edge designs of four different silicone hydrogel lenses with the ocular surface
}

\author{
This article was published in the following Dove Press journal: \\ Clinical Ophthalmology \\ 25 May 2015 \\ Number of times this article has been viewed
}

\section{Semra Akkaya Turhan Ebru Toker}

Department of Ophthalmology, School of Medicine, University of Marmara, Istanbul, Turkey
Correspondence: Ebru Toker Department of Ophthalmology, School of Medicine, University of Marmara, Fevzi Çakmak Mah, Muhsin Yazıcıoğlu Cad, No 10 Üst Kaynarca, Pendik, Istanbul 34899, Turkey

Tel +905326946212

Email dretoker@gmail.com
Purpose: To evaluate the lens edge interaction with the ocular surface with different edge designs using optical coherence tomography and to examine the effect of lens power on the lens edge interactions.

Methods: Four types of silicone hydrogel lenses with different edge designs (round-, semiround-, chisel-, and knife-edged) at six different powers $(+5.0,+3.0,+1.0,-1.0,-3.0$, and -5.0 diopters) were fitted to both eyes of 20 healthy volunteers. Optical coherence tomography images were taken at the corneal center and at the limbus within 15-30 minutes after insertion. The images were evaluated with respect to two parameters: conjunctival indentation exerted by the lens edge; and the tear film gaps between the posterior surface of the lens and the ocular surface. The amount of conjunctival indentation was measured with the distortion angle of the conjunctiva at the lens edge.

Results: The degree of conjunctival indentation was highest with the chisel-edged design followed by the semi-round design $(P<0.0001)$. Knife- and round-edged lenses exerted similar levels of conjunctival indentation that was significantly lower compared to chisel-edged lens $(P<0.001)$. For each one of the tested lens edge designs, no significant difference was observed in the conjunctival indentation with respect to lens power. The chisel-edged lens produced the highest amount of conjunctival indentation for each one of the six lens powers $(P<0.0001)$. Postlens tear film gaps at the limbus were observed at most in the round-edge design $(P=0.001)$.

Conclusion: The fitting properties of contact lenses may be influenced by their edge design but not by their lens power.

Keywords: Acuvue Oasys, Air Optix Aqua, PremiO, PureVision 2 HD, silicone hydrogel contact lenses

\section{Introduction}

A comprehensive evaluation which includes patient-related factors (palpebral aperture, lid position, iris diameter, and corneal topography) and contact lens-related factors (fitting profile, lens movement after blink, and lens modulus) is the essential step in the contact lens fitting process. This will minimize the risk of future failures or problems because of poor lens fitting. Discontinuation of contact lens wear is a major problem in contact lens practice. Inappropriate lens fit is responsible for $7 \%$ of contact lens discontinuations. ${ }^{1}$ The primary reasons for discontinuation of contact lens wear over time have been reported to be discomfort, dryness, and red eyes. ${ }^{2,3}$ Comfort may be the most important factor in determining success versus failure of a contact lens 
wearer. Ocular discomfort, primarily caused by mechanical irritation linked to modulus, edge thickness, lens design, and inappropriate lens fit is the most important reason for discontinuation of contact lens wear. ${ }^{1,4,5}$ Modulus values represent lens stiffness and resistance to shape change. Higher modulus can be related to mechanical complications, including contact lens papillary conjunctivitis, superior epithelial arcuate lesions, limbal epithelial hypertrophy, contact lens-induced conjunctival staining (CLICS), and conjunctival epithelial flaps (CEFs). ${ }^{6-9}$

Slit-lamp examination is often used for evaluation of contact lens fit but it is not always sufficient to give information to evaluate the lens fitting relationship between a contact lens and the ocular surface, and it is often difficult to objectively quantify soft contact lens fitting. Successful lens fitting is affected by many factors, such as corneal shape, ${ }^{10}$ an adequate distribution of the contact lens weight over all the corneal surface, post-lens tear film, ${ }^{11,12}$ enough movement to provide an optimal tear turn-over, blink rate, ${ }^{13}$ and lens material and design, ${ }^{14}$ all of which provide minimal mechanical effect between the ocular surface and the contact lens.

Edge design is an extremely important and often underestimated parameter that can primarily affect comfort, lens positioning, lens motion, and overall fit. Both center thickness and edge thickness change with different lens powers.

Soft contact lenses cover the surface of the cornea and extend over the limbus onto the sclera by approximately $1 \mathrm{~mm}$, hence topographical features of the corneoscleral junction as well as the central and the peripheral cornea are important to evaluate this appropriate fitting relationship. Recent studies have shown that there is a smooth, gradual transition in topography between the cornea and the sclera having a tangential profile in the majority of cases, and have suggested that the interaction between the lens edge and the ocular surface at the periphery has an equal or more important role for lens fitting. ${ }^{15,16}$ Hence, determination of the topography of the peripheral cornea, corneoscleral junction, and the anterior sclera provides crucial information for proper lens fitting. ${ }^{15,17}$ Optical coherence tomography (OCT) may be suitable for this task. Anterior segment OCT can image the entire ocular surface and the contact lens on the eye, obtain quantitative topography of the ocular surface, and generate thickness maps of the soft contact lenses. ${ }^{18}$ OCT device is a noninvasive technique that can be used to visualize tears around contact lenses and to image the interactions between the ocular surface and the contact lens edge. ${ }^{17,19,20}$ The indentation in the previous study was only subjectively graded in different lens edge design. ${ }^{17}$ However, to the best of our knowledge, measuring the angle of conjunctival indentation for determination of quantitative assessment of lens edge-ocular surface interaction and the effect of lens power on these fitting characteristics using OCT has not been studied previously.

The purpose of this study was to evaluate the edge fitting and the interaction with the ocular surface of four silicone hydrogel lenses (SiHy) with different edge designs and materials using OCT, and also to examine the effect of lens power on these fitting characteristics.

\section{Methods}

A prospective, randomized (random integer generator; www.random.org/integers), and clinical comparative study was approved by the Marmara University Ethical Committee. The study followed the tenets of the Declaration of Helsinki. Informed consent was obtained from all subjects.

Both eyes of 20 healthy volunteers were fitted with $\mathrm{SiHy}$. Slit-lamp examination was performed by the same examiner (SAT) to confirm the proper lens fitting, defined as having a good centration and enough movement (the post-blink movement within 0.2 to $0.4 \mathrm{~mm}$ ). Each subject was enrolled in the study if they were aged between 18 and 40 years, and were successfully fit with the study lenses. There was no evidence or history of ocular surgery, dry eye, use of any ophthalmic medication, or ocular inflammation. None of the subjects wore contact lenses before or between study visits. Participants underwent a full ocular assessment. The order of the lens and diopter insertion was randomized for each eye of the subjects. Four types of SiHy contact lenses with different edge designs (round-, semi-round-, chisel-, and knife-edged) at six different powers $(+5.0,+3.0,+1.0,-1.0,-3.0$, and -5.0 diopters) were fitted. The study consisted of four visits for each subject. Each lens type was worn on a different visit, separated by at least 1 day. Table 1 shows the characteristic features of SiHy contact lenses used in this study.

Imaging and measurements were performed by the RTVue OCT system (Optuvue, Inc., Fremont, CA, USA), which uses Fourier domain OCT technology. An 8 mm-wide scan was made on the horizontal meridian. Within 15-30 minutes after insertion, OCT images for the center, nasal, and temporal sides of the lens were obtained for each eye after a blink. ${ }^{17}$ Images taken from the temporal side of the lens were evaluated with respect to two parameters: conjunctival indentation by the lens edge; and the tear film gaps between the posterior surface of the lens and the ocular surface. All OCT images were taken in a consulting room with controlled temperature $\left(15^{\circ} \mathrm{C}-25^{\circ} \mathrm{C}\right)$ and humidity $(30 \%-50 \%)$. All subjects were 
Table I Characteristics of the contact lenses used in the study

\begin{tabular}{|c|c|c|c|c|}
\hline Characteristic & Acuvue Oasys & PremiO & Air Optix Aqua & PureVision 2 HD \\
\hline Manufacturer & Johnson \& Johnson & Menicon & Ciba Vision & Bausch \& Lomb \\
\hline Diameter (mm) & 14 & 14 & 14.2 & 14 \\
\hline Base curvature (mm) & 8.4 & 8.6 & 8.6 & 8.6 \\
\hline Material & Senofilcon A & Asmofilcon A & Lotrafilcon B & Balafilcon A \\
\hline Modulus (MPa) & 0.72 & 0.9 & 1.2 & I.I \\
\hline Water content (\%) & 38 & 40 & 33 & 36 \\
\hline Center thickness ( -3.00 diopters) & 0.07 & 0.08 & 0.08 & 0.07 \\
\hline Edge shape & Knife-edged & Semi-rounded & Chisel-edged & Rounded \\
\hline Peripheral thickness (-3.00 diopters) & 0.07 & - & 0.12 & 0.07 \\
\hline Mid-peripheral thickness (-3.00 diopters) & 0.19 & - & 0.19 & 0.17 \\
\hline
\end{tabular}

Notes: Bausch \& Lomb Incorporated, Bridgewater, NJ, USA; Ciba Vision, Duluth, GA, USA; Johnson \& Johnson, New Brunswick, NJ, USA; Menicon Co., Ltd. Nagoya, Japan.

scheduled after 10 am to avoid the edematous cornea and the alteration of the tear film induced by sleep. . $^{1721,22}$

The amount of conjunctival indentation was measured with the distortion angle of the conjunctiva at the lens edge. An imaginary straight line was drawn along the contour of the conjunctival surface at the limbus. Another line was drawn passing along the axis of the indented conjunctiva at the lens edge. Then the angle at the intersection of these two lines was measured by the angle measurement tool on the OCT instrument. The larger the angle, the greater the degree of conjunctival indentation (Figure 1).

Tear film gaps at the limbus were also evaluated, as previously described. ${ }^{17}$ The presence of these gaps was recorded for each study lens (Figure 2).

\section{Statistical analysis}

Data analysis was performed by means of SPSS for Windows, version 17.0 (SPSS Inc., Chicago, IL, USA). The nonparametric analysis of variance (Kruskal-Wallis) and Dunn's post hoc tests were used to determine significant differences of the measured conjunctival indentation among lens types and lens powers. The differences of occurrence frequency of tear film gaps among lenses were compared with chi-square test. Differences were considered to be statistically significant when the $P$-value was $<0.05$.

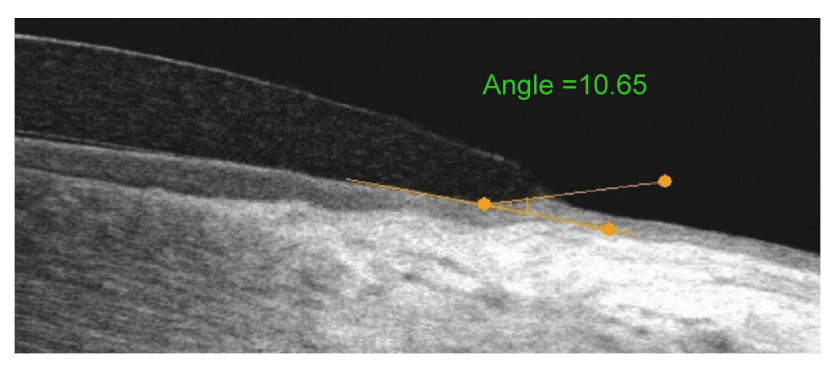

Figure I Measurement of conjunctival indentation.

\section{Results}

Forty eyes of 20 participants (ten women, ten men) with a mean age ( \pm standard deviation) of $25.1 \pm 3.8$ (range, 18-38) years were enrolled in this study. The average of keratometric values for right and left eyes were $43.73 \pm 1.69$ and $43.43 \pm 1.53$ diopters, respectively. Conjunctival indentation exerted by the lens edge for different edge designs are presented in Figure 3. When all data $(n=480)$ were examined regardless of lens power, conjunctival indentation was highest with the chisel-edge design followed by the semi-round-edge design $(P<0.0001)$ (Figure 4). There were no significant differences in the conjunctival indentation between the knife-edge and the round-edge designs $(P>0.05)$. The distribution of conjunctival indentation according to lens power is shown in Figure 5. The chisel-edged lens produced the highest amount of conjunctival indentation for each of the six lens powers $(P<0.0001)$. For each of the tested lens edge designs, no significant difference was observed in the conjunctival indentation with respect to lens power $(P>0.05)$. Post-lens tear film gaps at the limbus were present in $12 \%$ of all eyes. Post-lens tear film gaps at the limbus were observed at most with the round-edge design followed by the chisel-edge design (Figure 6). Post-lens tear

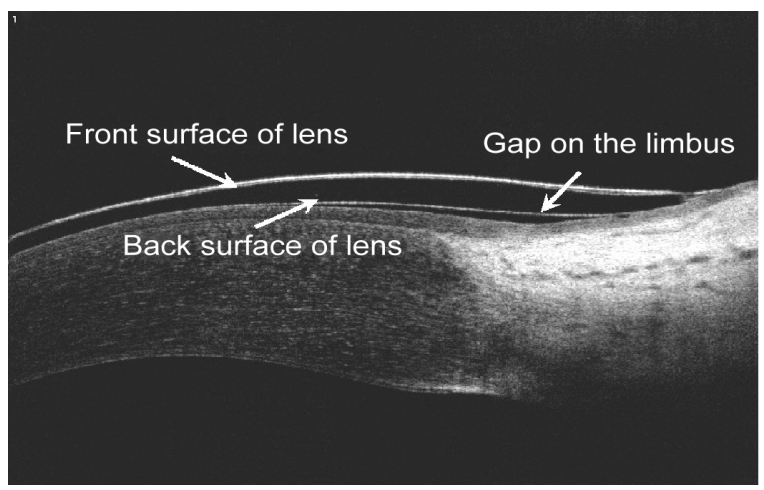

Figure 2 Visualization of limbal gaps located at the corneoscleral junction. 

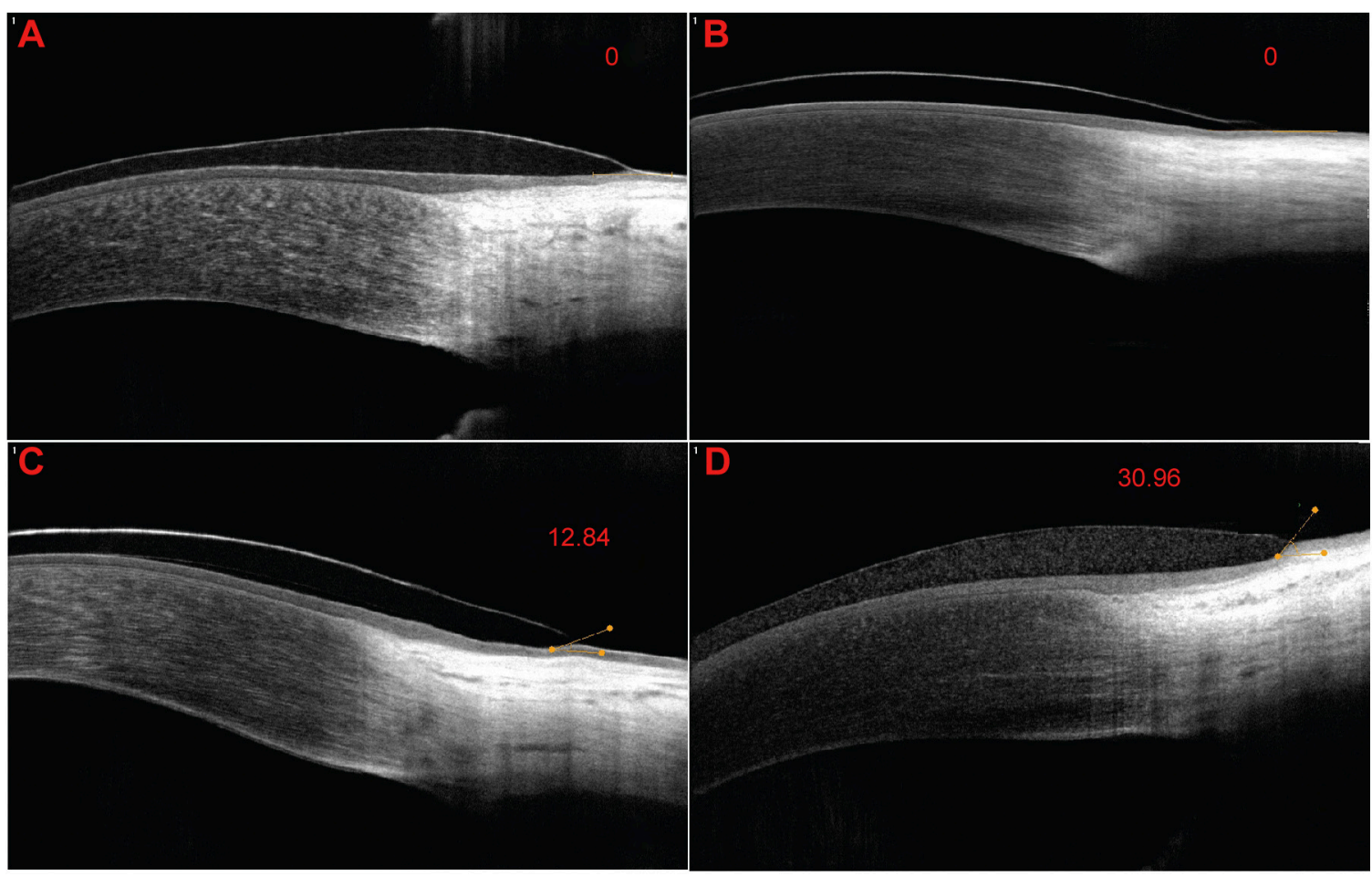

Figure 3 Angle of conjunctival indentation was measured by optical coherence tomography for different edge designs.

Notes: (A) Knife-edge design (Acuvue Oasys). (B) Round-edge design (PureVision 2 HD). (C) Semi-round edge design (PremiO). (D) Chisel-edge design (Air Optix Aqua).

\section{Conjunctival indentation}

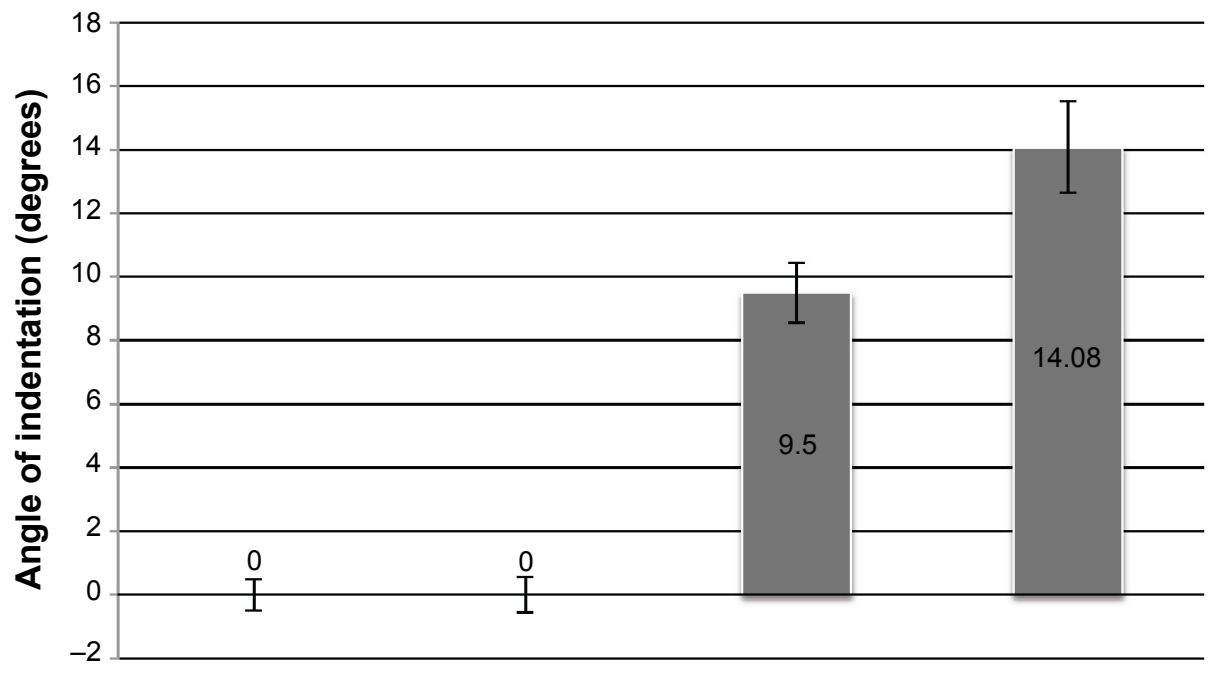

\begin{tabular}{lllll}
\hline & $\begin{array}{l}\text { Knife edge } \\
\text { (Acuvue } \\
\text { Oasys) }\end{array}$ & $\begin{array}{l}\text { Round edge } \\
\text { (PureVision } \\
\text { 2 HD) }\end{array}$ & $\begin{array}{l}\text { Semi-round edge } \\
\text { (PremiO) }\end{array}$ & $\begin{array}{l}\text { Chisel edge } \\
\text { (Air Optix Aqua) }\end{array}$ \\
\hline Median \pm SEM & $0.00 \pm 0.50$ & $0.00 \pm 0.55$ & $9.50 \pm 0.94$ & $14.08 \pm 1.44$ \\
Mean \pm SD & $4.12 \pm 5.54$ & $3.85 \pm 6.09$ & $10.43 \pm 10.34$ & $18.28 \pm 15.82$ \\
\hline
\end{tabular}

Figure 4 Comparison of conjunctival pressure exerted by the lens edge for different edge designs, regardless of the lens power. Abbreviations: SD, standard deviation; SEM, standard error of the mean. 


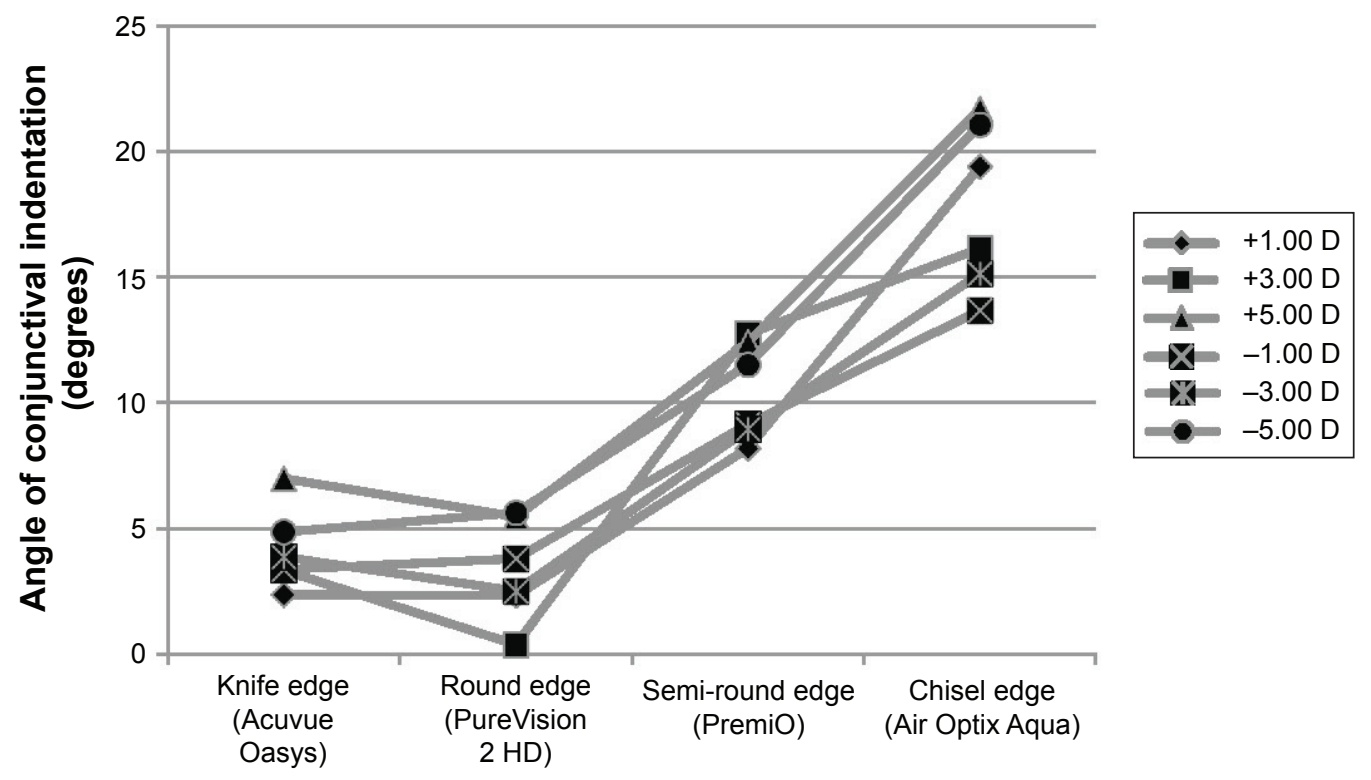

Figure 5 Distribution of conjunctival indentation according to lens power and edge design. Abbreviation: D, diopters.

film gaps at the limbus were observed mostly with plus-power lenses $(P=0.017)$ (Figure 7).

\section{Discussion}

Slit-lamp examination is the gold standard method in the evaluation of appropriate lens fit. Although this simple method provides proper assessment in the majority of patients, it gives limited information about the interaction between the ocular surface and the posterior surface of the contact lens. The posterior lens surface interacts with the cornea, limbus, and the bulbar conjunctiva, and the anterior surface has close relationship with the palpebral conjunctiva and upper/lower lid margins. So, a better understanding of this interaction will provide more valuable information to prevent complications associated with contact lens wear.

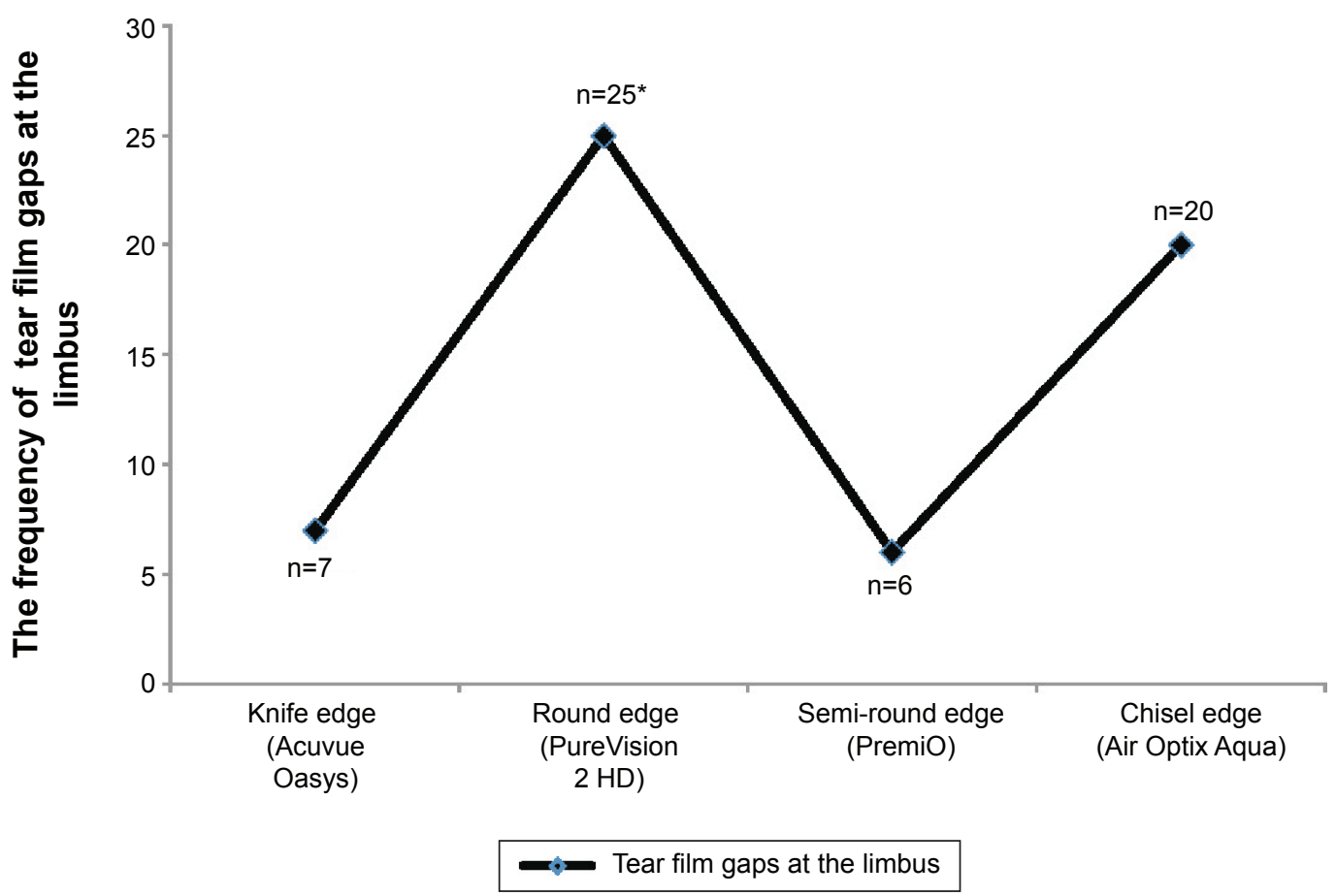

Figure 6 Occurrence of tear film gaps at the limbus according to lens edge designs.

Note: *Significantly different from knife edge $(P=0.00 \mathrm{I})$ and semi-round edge $(P=0.0004)$. 


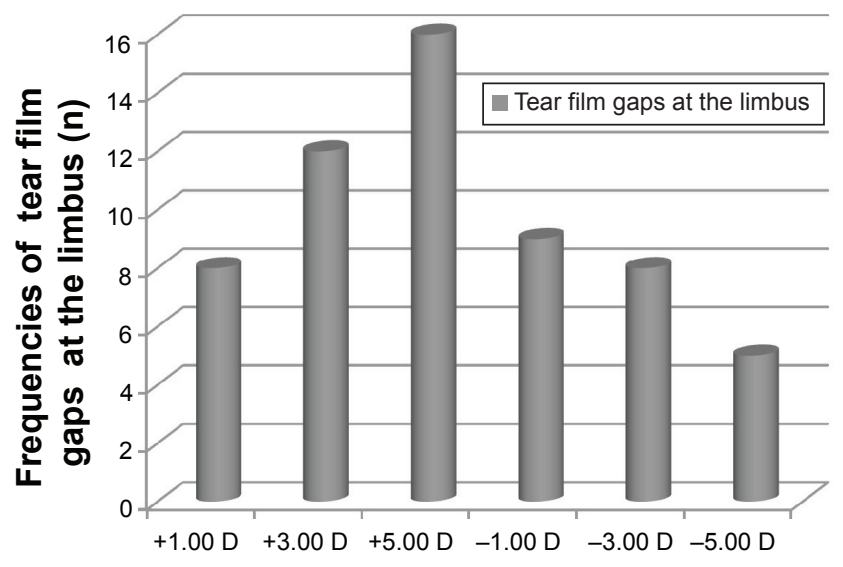

Figure 7 Distribution of limbal tear film gaps according to lens powers. Note: $P=0.017$.

Abbreviation: $\mathrm{D}$, diopters.

SiHy lens materials have a higher modulus than conventional hydrogel lenses and are more prone to induce mechanical complications. ${ }^{5}$ Two recently described clinical conditions related with mechanical interaction between the conjunctival tissue and the contact lens are CEFs and CLICS. ${ }^{5,8,23,24} \mathrm{CEFs}$ are mostly located at the superior and inferior quadrants of the bulbar conjunctiva adjacent to the lens edge and they are believed to occur due to repeated interaction of the lens periphery with the ocular surface causing delamination of the superficial layers of the conjunctival epithelium. Although $\mathrm{CEF}$ is an asymptomatic condition, its long-term risk for any inflammatory response is not yet clear. The occurrence of CEFs has been linked to modulus of rigidity of the lens material, edge design, and wearing modality. ${ }^{5}$ Previous clinical studies have suggested that chisel-edge-design SiHy lenses induce a higher incidence of CEFs than the round-edgedesign ones, ${ }^{5,23}$ and continuous wear modality is more likely to exacerbate this mechanical effect compared to daily wear. ${ }^{5}$ This implies that the management of CEFs may involve modifying the wearing modality and changing to a round-edge lens design. The other mechanical complication is CLICS, which is more common with certain lens edge configurations than others. Direct contact between the lens periphery and the ocular surface irritating the conjunctival epithelium causes staining at the limbal area. Maissa et $\mathrm{al}^{25}$ investigated the conjunctival staining produced in the circumlimbal region by SiHy lenses with different edge designs, and also the relationship between circumlimbal staining and comfort. They reported that round-edge design produced the lowest staining while knife-edge design, being in close apposition with the ocular surface, produced the highest. Balafilcon A and lotrafilcon B lenses have a similar material rigidity, but circumlimbal staining produced by a balafilcon A lens was lower than that produced by a lotrafilcon B lens. This finding suggested that the lens edge design is the primary factor in controlling circumlimbal staining. ${ }^{25}$ When the multifactorial comfort analysis was evaluated, the lens edge design was the primary influential factor that affected lens comfort: the knife-edge design had the best comfort; the round-edge design had the worst comfort. There was an inverse relationship between circumlimbal staining and lens comfort, and the authors concluded that CLICS was not a significant factor associated with decreased comfort. ${ }^{25}$ In our study, we observed the highest amount of conjunctival indentation with the chisel-edge design lens followed by the semi-round-edge design lens. Knife and round-edge designs induced the lowest amount of conjunctival indentation. In a recent study, Shen et a $\mathrm{l}^{17}$ used an analog scale $(0 \%, 25 \%, 50 \%, 75 \%, 100 \%)$ for indicating the degree of conjunctival buildup around the lens edge and compared four different types of soft contact lenses (two SiHy and two hydrogel contact lenses) with two different edge designs (rounded and angled). Similar to our results, they found no significant difference in the conjunctival buildup between the two SiHy lenses with rounded (PureVision; Bausch \& Lomb Incorporated, Bridgewater, NJ, USA) and angled-edge designs (Acuvue Advance; Vistakon, Johnson\&Johnson Vision Care, Inc., Jacksonville, FL, USA). However, there was a significant difference among lower modulus hydrogel lenses in that the knife-edged lens (Acuvue 2; Vistakon) favored less conjunctival indentation than the round-edged one (Biomedics 55; CooperVision, CooperVision Inc., Fairport, NY). Wolffsohn et a ${ }^{26}$ investigated four experimental lens designs with the same material (senofilcon A) to evaluate the interaction of mid-peripheral lens shape profile ( $8.4 \mathrm{~mm}$ base curve and $8.8 \mathrm{~mm}$ base curve) and lens edge design (knife-edge design and chisel-edge design) with the ocular surface using OCT. They reported that epithelial indentation did not change with mid-peripheral lens shape profile or with edge design after optical distortion was corrected, unlike the findings of Shen et $\mathrm{al}^{17}$ and our study.

The present study was the first attempt to demonstrate the effect of lens power on lens fitting characteristics using OCT. In contrast to our presumption that high-minus-power lenses with thicker lens edges would indent conjunctiva more, lens power, regardless of lens edge design, was observed to have no significant influence on the conjunctival indentation exerted by the lens edge.

The dynamic interaction between the contact lens and the tear film influences several aspects of ocular physiology as well as vision and comfort. Tear exchange is of key interest in providing essential supplies to the tissues under the contact 
lens and in the removal of deposits, and the tear film layer is also important to reduce the friction between the ocular surface and the lens. The edge design may play an important role in the circulation of the tear fluid under the lens, and in the movement of the lens over the conjunctiva and the cornea. A higher level of the conjunctival buildup at the lens edge has a negative effect on tear exchange beneath the lens that causes tear film gaps between the contact lens and the ocular surface. ${ }^{17}$ The presence of gaps between the lens and the cornea may also be an indicator of how the contact lens conforms to the surface of the cornea. In our study, greater frequencies of tear film gaps at the limbus occurred with the round-edge followed by the chisel-edge-design lenses, with both having a higher modulus than the other two edge designs. This finding is in agreement with data previously reported by Shen et $\mathrm{al}^{17}$ who stated that the round-edged lenses (Biomedics 55 and PureVision) produced a higher percentage of tear film gaps on the cornea than the angle-edged lenses (Acuvue 2 and Acuvue Advance), and round-edged lenses (PureVision) had the highest percentage of tear film gaps at the limbus. This shows that the occurrence of tear film gaps between the lens and the cornea is associated not only with the edge configuration but also with the modulus of the material. SiHy lenses with a higher modulus may not conform to the shape of the eye compared to SiHy lenses with a lower modulus. As a consequence, the "stiffer" material does not drape over the cornea easily. ${ }^{27}$

Tear exchange under the contact lens is likely to be impacted by lens power besides the modulus of the material and the edge configuration as both center thickness and edge thickness change with different lens powers. In this study, limbal tear film gaps were mostly observed with plus-power lenses. The lens edge thickness in plus-power lenses is thinner than the apex. According to a recent study, touch points between the lens and the ocular surface were observed at the corneal apex, at the mid-periphery of the cornea and the limbus. ${ }^{17}$ Hence, these touch points and thickness differences between lens powers may have an impact on the circulation of the tear film, resulting in entrapment of tears in the form of tear film gaps. The dynamics of lens movement may also influence the tear exchange. ${ }^{28}$ Confirmation of these hypotheses needs to be elucidated in further studies.

In summary, this was the first study to demonstrate the quantitative assessment of the conjunctival indentation and to determine the effect of lens power on the lens fitting and tear exchange using OCT. Our results suggest that the fitting properties of contact lenses may be influenced by their edge design but not by lens power. Anterior segment OCT is a useful method to evaluate the fitting characteristics of contact lenses and can be used to show lens edge and ocular surface interaction. OCT findings of lens fit can be evaluated in further studies with respect to subjective comfort scores, clinical findings, and the lens movements.

\section{Acknowledgment}

This work has previously been presented at 41st ECLSO Congress, Istanbul, Turkey on 9-11 September 2011. Best oral presentation award was attributed to this paper.

\section{Disclosure}

The authors report no conflicts of interest in this work.

\section{References}

1. Young G. Why one million contact lens wearers dropped out. Cont Lens Anterior Eye. 2004;27(2):83-85.

2. Young G, Veys J, Pritchard N, Coleman S. A multi-centre study of lapsed contact lens wearers. Ophthalmic Physiol Opt. 2002;22(6):516-527.

3. Pritchard N, Fonn D, Brazeau D. Discontinuation of contact lens wear: a survey. Int Contact Lens Clin. 1999;26(6):157-162.

4. Begley CG, Caffery B, Nichols KK, Chalmers R. Responses of contact lens wearers to a dry eye survey. Optom Vis Sci. 2000;77(1):40-46.

5. Santodomingo-Rubido J, Wolffsohn J, Gilmartin B. Conjunctival epithelial flaps with 18 months of silicone hydrogel contact lens wear. Eye Contact Lens. 2008;34(1):35-38.

6. Skotnitsky CC, Naduvilath TJ, Sweeney DF, Sankaridurg PR. Two presentations of contact lens-induced papillary conjunctivitis (CLPC) in hydrogel lens wear: local and general. Optom Vis Sci. 2006;83(1):27-36.

7. Dumbleton K. Adverse events with silicone hydrogel continuous wear. Cont Lens Anterior Eye. 2002;25(3):137-146.

8. Graham AD, Truong TN, Lin MC. Conjunctival epithelial flap in continuous contact lens wear. Optom Vis Sci. 2009;86(4):e324-e331.

9. Synder C. Modulus and its effect on contact lens fit. Contact Lens Spectrum. 2007;22(2).

10. Young G, Schnider C, Hunt C, Efron S. Corneal topography and soft contact lens fit. Optom Vis Sci. 2010;87(5):358-366.

11. Little SA, Bruce AS. Hydrogel (Acuvue) lens movement is influenced by the postlens tear film. Optom Vis Sci. 1994;71(6):364-370.

12. Little SA, Bruce AS. Postlens tear film morphology, lens movement and symptoms in hydrogel lens wearers. Ophthalmic Physiol Opt. 1994;14(1):65-69.

13. Golding TR, Bruce AS, Gaterell LL, Little SA, Macnamara J. Soft lens movement: effect of blink rate on lens settling. Acta Ophthalmol Scand. 1995;73(6):506-511.

14. Tranoudis I, Efron N. In-eye performance of soft contact lenses made from different materials. Cont Lens Anterior Eye. 2004;27(3):133-148.

15. Hall LA, Young G, Wolffsohn JS, Riley C. The influence of corneoscleral topography on soft contact lens fit. Invest Ophthalmol Vis Sci. 2011;52(9):6801-6806.

16. Meier D. Das corneo-skleral-profile-ein kriterium individueller kontactlinsenanpassung [The corneo-scleral profile - a criterion of individual contact lens fitting]. Die Kontaklinse. 1992;26(4):4-11. German.

17. Shen M, Cui L, Riley C, Wang MR, Wang J. Characterization of soft contact lens edge fitting using ultra-high resolution and ultra-long scan depth optical coherence tomography. Invest Ophthalmol Vis Sci. 2011;52(7):4091-4097.

18. Shen M, Wang MR, Wang J, Yuan Y, Chen F. Entire contact lens imaged in vivo and in vitro with spectral domain optical coherence tomography. Eye Contact Lens. 2010;36(2):73-76. 
19. Wang J, Jiao S, Ruggeri M, Shousha MA, Chen Q. In situ visualization of tears on contact lens using ultra high resolution optical coherence tomography. Eye Contact Lens. 2009;35(2):44-49.

20. Kaluzny BJ, Kaluzny JJ, Szkulmowska A, et al. Spectral optical coherence tomography: a new imaging technique in contact lens practice. Ophthalmic Physiol Opt. 2006;26(2):127-132.

21. Patel S, Bevan R, Farrell JC. Diurnal variation in precorneal tear film stability. Am J Optom Physiol Opt. 1988;65(3):151-154.

22. Shen M, Wang J, Qu J, et al. Diurnal variation of ocular hysteresis, corneal thickness, and intraocular pressure. Optom Vis Sci. 2008; 85(12):1185-1192.

23. Løfstrøm T KA. A conjunctival response to silicone lens wear: a new finding reveals how silicone hydrogel lenses may affect the conjuctival epithelium. Contact Lens Spectrum. 2005;20(9):42-44.

24. Lin MC, Yeh TN. Mechanical complications induced by silicone hydrogel contact lenses. Eye Contact Lens. 2013;39(1):115-124.
25. Maissa C, Guillon M, Garofalo RJ. Contact lens-induced circumlimbal staining in silicone hydrogel contact lenses worn on a daily wear basis. Eye Contact Lens. 2012;38(1):16-26.

26. Wolffsohn JS, Drew T, Dhallu S, Sheppard A, Hofmann GJ, Prince M. Impact of soft contact lens edge design and midperipheral lens shape on the epithelium and its indentation with lens mobility. Invest Ophthalmol Vis Sci. 2013;54(9):6190-6197.

27. Dumbleton KA, Chalmers RL, McNally J, Bayer S, Fonn D. Effect of lens base curve on subjective comfort and assessment of fit with silicone hydrogel continuous wear contact lenses. Optom Vis Sci. 2002; 79(10):633-637.

28. Cui L, Shen M, Wang MR, Wang J. Micrometer-scale contact lens movements imaged by ultrahigh-resolution optical coherence tomography. Am J Ophthalmol. 2012;153(2):275-283.e271.
Clinical Ophthalmology

\section{Publish your work in this journal}

Clinical Ophthalmology is an international, peer-reviewed journal covering all subspecialties within ophthalmology. Key topics include: Optometry; Visual science; Pharmacology and drug therapy in eye diseases; Basic Sciences; Primary and Secondary eye care; Patient Safety and Quality of Care Improvements. This journal is indexed on

\footnotetext{
Submit your manuscript here: http://www.dovepress.com/clinical-ophthalmology-journal
}

\section{Dovepress}

PubMed Central and CAS, and is the official journal of The Society of Clinical Ophthalmology (SCO). The manuscript management system is completely online and includes a very quick and fair peer-review system, which is all easy to use. Visit http://www.dovepress.com/ testimonials.php to read real quotes from published authors. 